\title{
HIPERTENSIÓN EN EL PERSONAL DE LA UNIVERSIDAD ESTATAL A DISTANCIA DE COSTA RICA
}

\author{
MS.O. Lourdes Arce Espinoza ${ }^{1}$, M.Sc. Julián Monge-Nájera ${ }^{2}$ \\ ${ }^{1}$ Enfermera, Servicios Médicos, UNED, larce@uned.ac.cr \\ ${ }^{2}$ Investigador, Vicerrectoría Investigación, UNED, julianmonge@gmail.com \\ San José, Costa Rica \\ 2009
}

RESUMEN

\begin{abstract}
La prevalencia de la Hipertensión Arterial (HTA) se ha incrementado en todo el mundo y las acciones preventivas resultan insuficientes, ya que solamente un tercio total de esta población está controlada. La HTA es la primera causa de morbilidad y mortalidad mundial. En este artículo se presenta el primer estudio sobre los niveles de hipertensión en el personal de una universidad a distancia, mediante el análisis del total de consultas en la Universidad Estatal a Distancia de Costa Rica al 15 de diciembre del 2007 (1526 expedientes médicos). La población estudiada se encuentra en edades comprendidas entre los 20 a 70 años, residentes del Gran Área Metropolitana, con nivel socioeconómico y preparación académica variable. Se analizó la relación entre la HTA y las variables tratamiento, sexo, edad y tipo de trabajo, usando la prueba chi-cuadrado. Solo 45 de los pacientes conocían de su problema de hipertensión antes de llegar a consulta en el servicio médico institucional y 136 pacientes reciben tratamientos que incluyen Enalapril e Hidroclorotiazida. La cantidad de pacientes hipertensos es máxima entre quienes tienen más de 20 años de trabajar en la institución, especialmente si ocupan altos puestos. No hubo marcadas diferencias entre mujeres y hombres. Se concluye que la existencia de un servicio médico interno ha permitido que la población de la universidad mantenga controlada satisfactoriamente su presión arterial.
\end{abstract}

PALABRAS CLAVE: hipertensión, consulta externa, educación a distancia, personal de universidad, Costa Rica

\begin{abstract}
The prevalence of Arterial Hypertension (AHT) has increased worldwide and preventive measures are insufficient since only one third of the population is being treated. AHT is the primary cause of morbidity and mortality in the world. In this article is presented the first study on hypertension levels of personnel of a Distance Education university based on the analysis of all medical consultations in the Costa Rican State University for Distance Education (Universidad Estatal a Distancia-UNED) as of December 15, 2007 (1,526 medical files). The population studied ranges from 20 to 70 years of age and is comprised of residents of the Greater Metropolitan Area (Costa Rica) with varied socioeconomic and academic levels. The Statgraphics Centurion $X V$ software and the chi-square test were used to analyze variables such as treatment administered, sex, age, and type of work. Only 45 patients knew that they suffered from hypertension prior to their consultation with the university medical service and 136 were treated with Enalapril and Hydrochlorothiazide. The number of hypertensive patients is higher among those who have worked at the institution for more than 20 years, especially in those holding higher positions. No marked differences were found between men and women. It is concluded that the existence of a university medical service has permitted faculty and staff to satisfactorily control their blood pressure.
\end{abstract}

KEY WORDS: Hypertension, outpatient service, distance education, university personnel, Costa Rica.

\section{Introducción}


La Hipertensión Arterial (HTA) es una enfermedad crónica generalmente asintomática, se caracteriza por la elevación de la presión arterial sistólica (PAS) mayor a $120 \mathrm{~mm} \mathrm{Hg} \mathrm{y/o}$ diastólica (PAD) mayor a $80 \mathrm{~mm} \mathrm{Hg}$, su prevalencia se ha incrementado en todo el mundo entre un 3\% a un 18\%, entre los años 1999-2002. Los programas y las acciones preventivas resultan insuficientes, ya que solamente un tercio de esta población está controlada (Haijar y Kotchen, 2006).

La HTA es la primera causa de morbilidad y mortalidad mundial, con 7.1 millones de fallecidos en el 2006 (Holguin, Correa, Arrivillaga, Cáceres y Varela, 2006). Su causa es la sumatoria de los factores de riesgos modificables (exceso de peso, ingesta de sodio por encima de 1.5 gramos al día, consumo de alcohol, inactividad física, fumado y dieta ricas en grasas) y no modificables (género, etnia, edad y herencia) (Huerta, 2001; Vásquez, Fernández, Álvarez, Roselló y Pérez, 2006; Li et ál., 2005).

La presencia de HTA, en conjunto con la obesidad, las dislipidemias y la resistencia insulínica, se conoce como síndrome metabólico (SM). Los trastornos metabólicos y la asociación entre HTA han sido ampliamente descritos en los últimos 10 años, así como su aumento en el riesgo de otras complicaciones coronarias y posible mortalidad, producto de diferentes combinaciones de sus factores de riesgo (Jarvis et ál., 2007; Gao, Nelson y Tucker, K. 2007; Xiang, Nelson, y Tucker, 2007).

Estadísticamente, se ha podido comprobar que las mujeres presentan mayor control de la HTA que los hombres (Plans, Tesserras, Pardell y Salleras, 2002); y que con la edad incrementan las cifras entre 20 a $30 \mathrm{~mm} \mathrm{Hg}$, entre pacientes de 40 años y los mayores de 60 años (sean estos normotensos o hipertensos). A partir de los 70 años de edad, los pacientes normotensos tienen un riesgo de un $90 \%$ de desarrollar hipertensión debido a la rigidez de los vasos sanguíneos (Vásquez et ál., 2006).

Existe un 16\% de hipertensos no diagnosticados, principalmente porque no cuentan con acceso a los servicios de salud, su baja escolaridad (estas personas son incapaces de reconocer los factores de riesgo de la HTA) y la falta de síntomas (Méndez y Rosero, 2007). Además, las creencias populares acerca de los tratamientos o de cómo evitar esta patología contribuyen de manera significativa a las bajas tasas de control (Wilson y colaboradores, 2002). Los aspectos culturales y los estilos de vida siguen siendo un problema común y contribuyen a la morbi-mortalidad de la HTA (Han, Kim, Kang, Kim y Kim, 2007).

Otro factor decisivo en la aparición de la HTA es el estrés. Algunas investigaciones sugieren que el estrés laboral contribuye de forma positiva a la aparición de HTA, debido a que produce alteración en los procesos fisiológicos normales de recuperación y descanso (Yang, Schnall, Jáuregui, Su y Baker, 2006). Aunque diversos estudios epidemiológicos ligan al estrés laboral con la aparición de HTA, independientemente de la ocupación, se ha determinado también que esta aseveración requiere de más estudios (Rose, Newman, Bennet y Tyroler, 1999, Rubio, Vallejo y Martínez, 2000).

La herencia o historia familiar es un factor determinante en la aparición de la HTA (Katzmarzyk, Rankinen, Perusse, Rao y Bouchard, 2001; Goldstein, Shapiro y Guthrie, 2006). Además, donde no existe historia familiar de HTA, algunos autores consideran que la familia puede convertirse en una fuente de estrés, produciendo efectos nocivos a largo plazo y por ende el desarrollo de HTA. Por medio de la herencia o del estrés producido, la familia 


\section{MIHSalud}

URL www.una.ac.cr/mhsalud

podría ser responsable de una alto porcentaje de riesgo en la aparición de la HTA (Huerta, Bautista, Irigoyen y Arrieta, 2005).

Algunos estudios realizados en niños y adolescentes con historia familiar de HTA, reconocen que pese a que las mediciones se encontraban dentro de los parámetros normales, su variabilidad sugiere que la historia familiar es un factor de riesgo importante y por lo tanto de importancia en la aparición de HTA en la adultez (Lawlor et ál., 2005; Borges, Peres y Horta, 2007; Barton, Gilbert, Baramme y Granger, 2006; Hulanicka, Lipowicz, Koziel y Kowalisko, 2007).

El estrés, la ira, la ansiedad y la depresión tienen una fuerte relación estadística con la HTA (Olmos et ál., 1999; Jhalani et ál., 2005; Yan et ál., 2006). Los factores de riesgo son los mismos en todas las etnias, sin embargo la agresividad con que se presenta la patología es mayor en pacientes de origen africano. (Hayes et ál., 2003; Holmes, Arispe y Moy, 2005; Higginbottom, 2006).

En Costa Rica la hipertensión arterial constituye una de las causas más frecuentes en las consultas médicas. Es una de las patologías con el costo más alto en la consulta externa; se espera que para el 2060 la población de adultos mayores de Costa Rica haya aumentado a 2 millones de personas y con ello las enfermedades crónicas, así como el costo en su atención médica (Méndez y Rosero, 2007).

Este trabajo presenta el primer estudio sobre los niveles de hipertensión en el personal de una universidad a distancia; y analiza la relación del sexo, la edad y el tipo de trabajo efectuado, con base en el personal de la Universidad Estatal a Distancia de Costa Rica, UNED, sobre la cual no existen estudios previos.

\section{Metodología}

\section{Instrumentos}

Expediente médico como fuente de información primaria

\section{Esfigmomanómetro TYCOS CERTIFIED}

Estetoscopio LITTMAN

Estos dos últimos para tomar la presión arterial.

\section{Sujetos}

Se incluyó a todos los pacientes de la consulta externa del servicio médico de la UNED que consultaron por HTA entre el 14 de enero de 2002 y el 14 de diciembre de 2007, para un total de 179 pacientes hipertensos, 95 mujeres y 84 hombres.

La población estudiada tiene entre 20 y 70 años (media: 22.3 años) y son residentes del Gran Área Metropolitana de Costa Rica, con nivel socioeconómico y preparación académica variable, ya que este estudio abarcó el personal de apoyo (como los conserjes), académicos (con estudios superiores de maestrías y doctorados), jefaturas y otros altos niveles administrativos. Estado civil: 140 pacientes casados, 29 en unión libre y 10 solteros. La mayor parte de la población tiene entre 6 meses y 30 años de laborar en esta institución (Archivo del Servicio Médico UNED). 


\section{Procedimientos}

La recolección de los datos desde la fuente primaria de información se realizó en dos etapas: la primera comprendió la revisión del expediente médico (total 1526), para determinar cuáles pacientes habían consultado por HTA, encontrándose un total de 179. La segunda etapa fue la digitación de los datos en un archivo Excel. A partir de este registro se procedió a la codificación de los datos y a su análisis estadístico (ver apartado sobre estadística).

\section{Aspectos éticos}

Este estudio cuenta con la aprobación de la Vicerrectoría de Investigación de la UNED, que es la instancia correspondiente y vela por los aspectos éticos y administrativos de las investigaciones realizadas en la institución. Al tratarse de un análisis a posteriori de datos estadísticos institucionales, en los cuales ni se somete a los pacientes a un tratamiento experimental ni se usan datos individualizados (se trata de un análisis semejante a las estadísticas institucionales que publica la Caja Costarricense de Seguro Social), no corresponde solicitar el consentimiento informado de los pacientes.

\section{Análisis estadístico}

El Programa Excel y el Programa estadístico Statgraphics Centurion XV se utilizaron para agrupar y analizar los datos. Se utilizó una metodología descriptivo-cuantitativa y de prueba de hipótesis; la primera para presentar la distribución de pacientes según sexo, edad, tratamiento y tipo de trabajo efectuado. Para las pruebas de hipótesis se usó Chi-cuadrado de Contingencia, la cual mide asociación entre variables, y en el caso de la interrelación edad, sexo, puesto e hipertensión, también se utilizó una regresión m. Y el 0,05 como valor de rechazo para las hipótesis.

\section{Resultados}

De las 1526 personas con expediente, 179 han consultado por HTA (12 \%).

Distribución por género del personal de la Universidad Estatal a Distancia de Costa Rica y antecedentes de Hipertensión Arterial

Solo 45 pacientes conocían de su problema de HTA antes de llegar a consulta en el servicio médico institucional, y la proporción no difiere entre mujeres y hombres $\left(X^{2}=0.05\right.$, grados de libertad=1, p=0.8231; Gráfico 1).

\section{Gráfico 1. Distribución por sexo del personal de la Universidad Estatal a Distancia y antecedentes de Hipertensión Arterial}




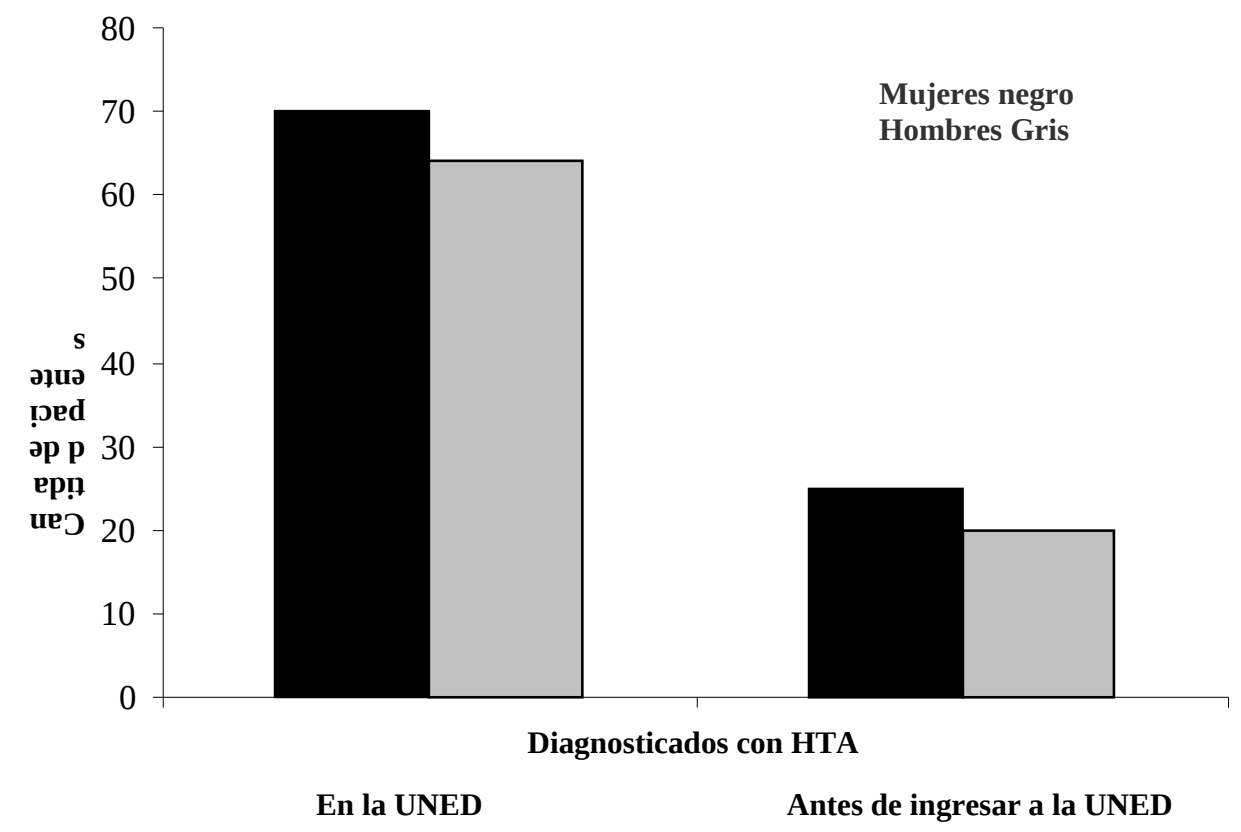

Fuente: Archivo de Servicio Médico UNED, 2007

\section{Terapia antihipertensiva}

Los tratamientos que se administran son de una compleja variedad. Del total de pacientes, 69 hombres y 67 mujeres reciben tratamientos que incluyen Enalapril, Hidroclorotiazida o su combinación. Los restantes, 26 mujeres y 17 hombres, reciben otros tratamientos (Tabla $1)$.

Tabla 1. Distribución de la terapia antihipertensiva en el personal de la Universidad Estatal a Distancia de Costa Rica 


\begin{tabular}{lcc}
\hline \multicolumn{1}{c}{ Tratamiento } & Femenino & Masculino \\
Enalapril & 22 & 37 \\
Enalapril + Hidroclorotiazida O Tritace + & & \\
Hidroclorotiazida & 12 & 14 \\
Amlodipina & 8 & 6 \\
Atenolol/ Propanolol & 9 & 6 \\
Hidroclorotiazida/ Furosemida & 5 & 1 \\
Irbesartan/Losartan/Aprovel & 8 & 1 \\
Atenolol/Propanolol + Hidroclorotiazida O & & \\
Ziac & 9 & 3 \\
Otras combinaciones & 22 & 16 \\
\hline
\end{tabular}

Fuente: Archivo de Servicio Médico UNED, 2007

La mayoría de pacientes masculinos no recibieron tratamiento con betabloqueadores, el cual está asociado a disfunción eréctil. Reciben una proporción ligeramente mayor que las mujeres de Enalapril e Hidroclorotiazida, pero en este aspecto la diferencia entre mujeres y hombres está en el borde de lo significativo (Prueba Exacta de Fisher, p=0,0497; datos agrupados en dos categorías por requisitos de la prueba: 1=Enalapril, Hidroclorotiazida o ambas; 2=Otros. Gráfico 2).

\section{Gráfico 2. Distribución por sexo del tratamiento prescrito del personal de la Universidad} Estatal a Distancia de Costa Rica 


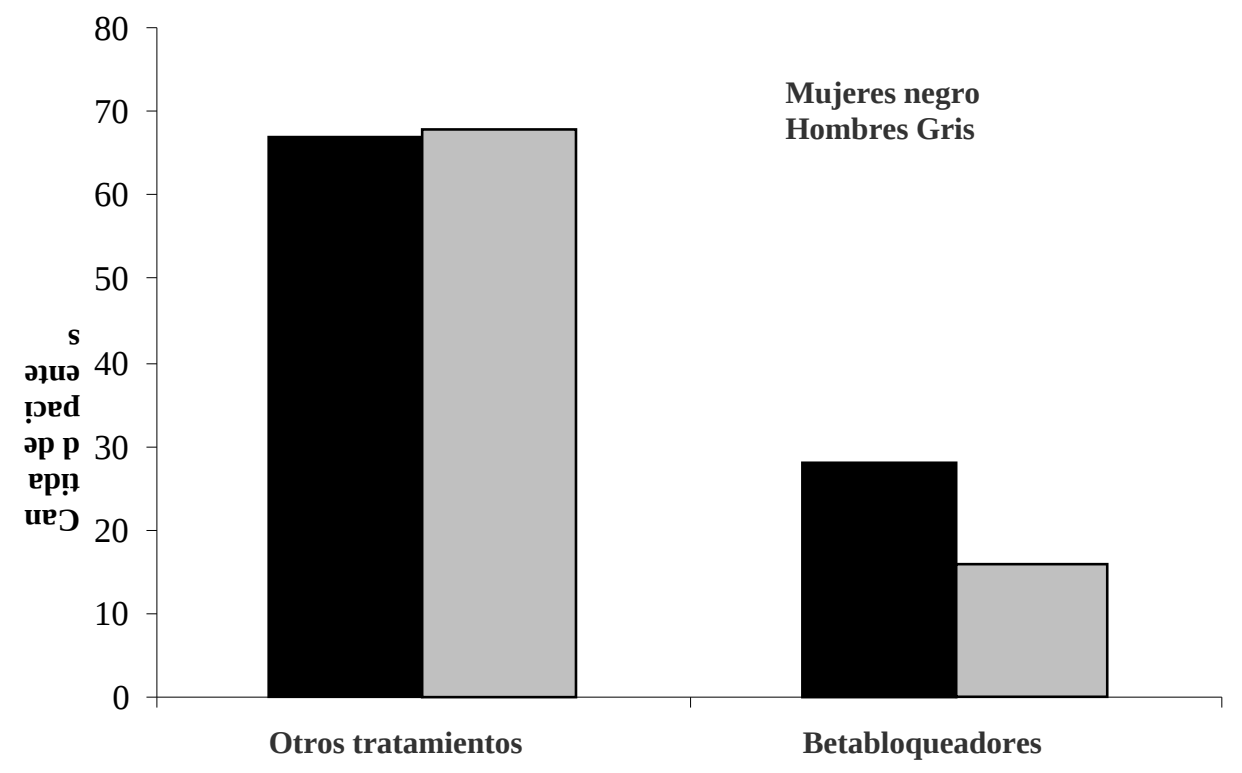

Tipo de tratamiento

Fuente: Archivo de Servicio Médico UNED, 2007

Relación entre antigüedad laboral, edad, sexo e hipertensión en la Universidad Estatal a Distancia de Costa Rica

El 24\% de los pacientes con más de 20 años de trabajar en la institución son hipertensos, valor similar al de quienes tienen menos de 10 años (23\%). En cambio, la tasa de hipertensión no supera el $8 \%$ en quienes tienen 11-20 años de trabajar en la UNED (Tabla 2). La mayoría de los pacientes hipertensos tiene más de 40 años de edad. No hay diferencia entre mujeres y hombres (Tabla 2$)\left(\mathrm{X}^{2}=1.7\right.$, grados de libertad=2, $\left.\mathrm{p}=0.4274\right)$.

Tabla 2. Número de pacientes con hipertensión arterial, distribuidos por antigüedad laboral, edad y sexo, de la Universidad Estatal a Distancia de Costa Rica 


\begin{tabular}{ccc}
\hline Antigüedad (años cumplidos) & $\begin{array}{l}\text { Mujeres } \\
\text { \% }\end{array}$ & Hombres \% \\
\hline $0-10$ & 23 & 20 \\
$11-20$ & 5 & 8 \\
$21-30$ & 24 & 20 \\
Edad (años cumplidos) & 3 & 3 \\
$20-30$ & 4.50 & 7.75 \\
$31-40$ & 20.70 & 16 \\
$41-50$ & 23 & 15 \\
$51-60$ & 2.30 & 5 \\
$61-70$ &
\end{tabular}

Fuente: Archivo de Servicio Médico UNED, 2007

Relación entre hipertensión arterial y ocupación del personal de la Universidad Estatal a Distancia de Costa Rica

La proporción de pacientes hipertensos se asocia con el tipo de ocupación (Tabla 3), ya que quienes ocupan jefaturas y otros altos puestos tienen mayor tasa de hipertensión $\left(X^{2}=6.76\right.$, grados de libertad $=4, p<0,05$ ). Esto es confirmado por una regresión múltiple que cruzó sexo, edad y puesto como posibles predictores de la hipertensión, la cual indicó que el puesto es la variable asociada $\left(R^{2}=0,0487 ; \mathrm{p} 0,001 ; \mathrm{N}=1526\right.$ pacientes).

Tabla 3. Número de pacientes con hipertensión, distribuidos por ocupación en el personal de la Universidad Estatal a Distancia de Costa Rica

\begin{tabular}{ccc}
\hline Ocupación & $\begin{array}{c}\text { Subtotal de pacientes y } \\
\text { porcentaje que son } \\
\text { hipertensos (\%) }\end{array}$ & $\begin{array}{c}\text { Total de empleados de la } \\
\text { UNED en esa categoría }\end{array}$ \\
\hline $\begin{array}{c}\text { Jefaturas y otros altos niveles } \\
\text { administrativos }\end{array}$ & $64(33 \%)$ & 192 \\
$\begin{array}{c}\text { Personal académico } \\
\text { Resto del personal } \\
\text { administrativo }\end{array}$ & $54(5 \%)$ & 1107 \\
\hline
\end{tabular}

Fuente: Archivo de Servicio Médico UNED, 2007

El 90\% de empleados de la UNED con hipertensión se controla la presión arterial por lo menos una vez al mes, cuando acuden a su control o bien cuando retiran el tratamiento antihipertensivo. Un 10\% se controla la presión arterial al menos dos veces por año, ya que llevan su control en otra institución de salud y solo se les toma la presión cuando consultan por otra causa (L. Arce, observación personal).

\section{Discusión}

\section{Distribución por sexo y antecedentes de hipertensión arterial}

El personal del Servicio Médico de la UNED detectó la mayoría de los casos de HTA, pues solamente 45 pacientes conocían su padecimiento antes de ingresar a laborar. Actualmente en la UNED se les continúa el control de esta patología. Esto demuestra una ventaja de prestar el servicio de salud en la institución: el fácil acceso permitió detectar la mayoría de los casos y tener un control de la población. 


\section{MHSalud}

URL www.una.ac.cr/mhsalud

Según la literatura, los datos epidemiológicos indican que el riesgo de padecer HTA es mayor en el hombre que en la mujer y que la edad de aparición de los síntomas de esta patología es a partir de los 45 años (hombre) y 55 años (mujer), aunque hay que recordar que algunos casos son asintomáticos y su diagnóstico se hace por medio del control de la PA (Plans et ál., 2002). En este estudio no hubo diferencias significativas en la tasa de HTA en mujeres y hombres. Tal vez las mujeres de la UNED acuden con mayor frecuencia por control de salud, planificación, control prenatal, etc., lo que aumenta la probabilidad de descubrir la patología en ellas y elevando su tasa registrada de HTA al mismo nivel que el de los hombres.

\section{Terapia antihipertensiva en mujeres y hombres}

Existen muchas combinaciones de tratamientos antihipertensivos, aplicados según la respuesta fisiológica al tratamiento, historial y valoración médica, mediante exámenes de gabinete y control de presión arterial antes de recetar la terapia antihipertensiva.

Según Grimm, Grandits, y Svendsen, (1996), la mayoría de los pacientes son tratados con Enalapril y diurético, o bien combinaciones terapéuticas, sin diferencias de prescripción entre ambos mujeres y hombres. Aunque se ha ligado el consumo de antihipertensivos, específicamente betabloqueadores como el propanolol y atenolol, a disfunción eréctil, existe controversia. Por ejemplo, el estudio TOMHS realizado en 1996 analizó 902 hipertensos que tomaban diuréticos, betabloqueantes, IECAS, alfabloqueantes o calcioantagonistas; tras cuatro años de seguimiento, la disfunción eréctil estuvo relacionada con la edad del paciente más que con el tipo de fármaco (Grimm, Grandits, y Svendsen, 1996). En la UNED solo un porcentaje muy pequeño de la población utiliza este medicamento y ninguno de los pacientes ha informado de este efecto secundario.

\section{Relación entre antigüedad laboral, edad y sexo}

Con la edad, las personas tienden a aumentar de peso; el envejecimiento y la pérdida de elasticidad de los vasos sanguíneos, agravada en las mujeres por la caída estrogénica (Molina, 2007) es coherente con el aumento de los casos de hipertensión a partir de los 40 años.

\section{Ocupación e hipertensión arterial}

A pesar de que la HTA está ligada a muchos factores modificables y no modificables, y es la sumatoria la que genera el desarrollo esta patología, el estrés que produce la complejidad de las funciones de las jefaturas (Olmos et ál., 1999, Jhalani et ál., 2005, Yan et ál., 2006), explica su mayor porcentaje de HTA en quienes ocupan puestos de jefatura en la UNED.

\section{Conclusiones}

Los resultados producen las siguientes conclusiones:

1. La existencia de un servicio médico institucional de fácil acceso, ha permitido detectar una cantidad muy importante de casos de hipertensión previamente desconocidos, y dar el tratamiento adecuado a los pacientes. 


\section{MIHSalud}

URL www.una.ac.cr/mhsalud

2. La mayoría de los pacientes recibe tratamiento con enalapril e hidroclorotiazida, y no se han reportado problemas de disfunción eréctil en los casos en que se usa betabloqueadores.

3. No hay un patrón definido de que la hipertensión aumente claramente con los años de servicio laboral.

4. Una proporción importante de pacientes con hipertensión son mujeres y hombres con más de 40 años de edad.

5. Quienes ocupan jefaturas presentan mayor proporción de casos con hipertensión.

\section{Agradecimientos}

Agradecemos a Carolina Morales y Daniel Villalobos su revisión de un borrador anterior.

\section{Referencias}

Barton, A. J., Gilbert, L., Baramme, J., y Granger, T. (2006). Cardiovascular risk in Hispanic and non Hispanic preschoolers. Nursing Research, 55, 172-179.

Borges, L. M., Peres, M. A., y Horta, B., L. (2007). Prevalence of high blood pressure among schoolchildren in Cuiaba, Midwestern Brazil. Revista de Saúde Pública, 41, 530-538.

Gao, X., Nelson, M. y Tucker, K. (2007). Television viewing is associated with prevalence of metabolic syndrome in hispanic elders. Diabetes Care, 30, 694-700.

Goldstein, I. B., Shapiro, D. y Guthrie, D. (2006). Ambulatory blood pressure and family history of hypertension in healthy men and women. American Journal of Hypertension, 19, 486-491.

Grimm, R. H., Grandits, G., y Svendsen, K. (1996). Sexual problems and antihypertensive drugs treatment: results of the Treatment of Mild Hypertension Study (TOMHS). Journal of Urology, 155, 634-644.

Haijar, I. K., y Kotchen, Ta. (2006). Hypertension: Trends in prevalence, incidence and control. Annual Review of Public Health, 27, 465-490.

Han, H. R., Kim, K. B., Kang, J. J., Kim, E. Y., y Kim, M. T. (2007). Knowledge, beliefs and behaviors about hypertension control among middle-aged Korean Americans with hypertension. Journal of Community Health, 32, 324-342.

Hayes, D. K., Denny, C. H., Keenan, N. L., Croft, J. B., Sundaram, A. A., y Greenlund, K. J. (2003). Racial/ethnic and socioeconomic differences in multiple risk factors for heart disease and stroke in women: Behavioral risk factor surveillance system. Journal of Women Health, 15, 1000-1008. 


\section{MIHSalud}

URL www.una.ac.cr/mhsalud

Higginbottom, G. M. (2006). Pressure of life: Ethnicity as mediating factor in mild-life and older people, experience of high blood pressure. Sociology of Health and Illness, 28, 583-610.

Holguin, L., Correa, D., Arrivillaga, M., Cáceres, D., y Varela, M. (2006). Treatment compliance in arterial hypertension:efficacy a biopsychosocial intervention program. Universitas Psychologica, .5, 535-548.

Holmes, J. S., Arispe, I. E., y Moy, E. (2005). Heart disease and prevention: Race and age differences in heart disease prevention, treatment and mortality. Medical Care, 43, 33-41.

Huerta, R. B. (2001). Factores de riesgo para la hipertensión arterial. Archivos de Cardiología de México, 7, 208-210.

Huerta, D., Bautista, L., Irigoyen, A., y Arrieta, R. (2005). Estructura familiar y factores de riesgo cardiovascular en pacientes con Hipertensión Arterial. Archivos de Medicina Familiar, 7, 87-92.

Hulanicka, B., Lipowicz, A., Koziel, S., y Kowalisko, A. (2007). Relationship between early puberty and the risk of hypertension, overweight at age 50: Evidence for a modified Barker Hypothesis among Polish youth. Economics and Human Biology, 5, 48-60.

Jhalani, J., Goyal, T., Clemow,L., Schwartz, J. E., Pickering, T. G., y Gerin, W. (2005). Anxiety and outcome expectations predict the white coat effect. Blood Pressure Monitoring, 10, 317-319.

Jarvis, C., Hayman, L. L., Braun, L., Schwertz, D., Ferrans, C., y Piano, M. (2007). Cardiovascular Risk Factors and Metabolic Syndrome in Alcohol- and NicotineDependent Men and Women. Journal of Cardiovascular Nursing, 22, 429-435.

Katzmarzyk, P. T., Rankinen, T., Perusse, L., Rao, D. D. C., y Bouchard, C. (2001). Familial risk of high blood pressure in the Canadian population. American Journal of Human Biology, 13, 620-625.

Lawlor, D, A., O’Callaghan, M. J., Mamun, A. A., Williams, G. M., Bor, W., y Najman, J. M. (2005). Socioeconomic position cognitive function and clustering of cardiovascular risk factors in adolescence: Findings from the Mater University Study of Pregnancy and its outcomes. Psychosomatic Medicine, 67, 862-868.

Li, W., Liu, L. S., Puente, J. G., Li, Y. S., Jiang, X. J., Jin, S. G., Ma, H., Kong, L. Z., Ma, L. M., He, X. Y., Ma, S. X., y Chen, C. M. (2005). Hypertension and health-related quality of life: an epidemiological study in patients attending hospital clinics in China. Journal of Hypertension, 23, 1667-1676.

Méndez, Ch. E., Rosero-Bixby, L. (2007). Prevalencia de hipertensión en adultos mayores de Costa Rica. Población y Salud de Mesoamérica, 5, 1-9. 
Molina, D. R. (2007). Manual de Hipertensión Arterial en la Práctica Clínica de Atención Primaria: Factores que influyen en su prevalencia. Revista de la Sociedad Andaluza de Medicina Familiar, 14, 1-4.

Olmos, O., Coromina, E., Morales, M., Torres, P., Coviello, A., y Grosse, A. (1999). El examen oral como prueba de estrés en una población de estudiantes y su incidencia sobre la presión arterial. Revista de la Federación de Argentina de Cardiología, 28, 87-90.

Plans, P., Tesserras, R., Pardell. H., y Salleras, L. (2002). Epidemiología de la hipertensión arterial en la población adulta de Cataluña, España. Revista Médica Clínica, 98, 369-372.

Rose, K. M., Newman, B., Bennet, T., y Tyroler, H. A. (1999). The association between extent of employment and hypertension among women participants of the second national health and nutrition survey. Women and Health, 29, 13-29.

Rubio, C., Vallejo, D., y Martínez, F. (2000). Cardiología y Medicina del Trabajo: Un enfoque sobre factores condicionantes en el desarrollo de ciertas cardiopatías. Revista Medicina del Trabajo, 1, 75-82.

Vásquez, A., Fernández, M., Álvarez, N., Roselló, Y., y Pérez, M. (2006). Percepción de la Hipertensión como factor de riesgo, "Aporte del día mundial de lucha contra la hipertensión”. Revista Cubana Médica, 45. 1-10.

Wilson, R. P., Freeman, A., Kazda, M. J., Andrews, T. C., Berry, L., Vaeth, P. A. C., Victor, R. G. (2002). Lay beliefs about high blood pressure in a low to middle income urban African-American community: An opportunity for improving hypertension control. American Journal of Medicine, 112, 26-30.

Xiang, G., Nelson, M. E., y Tucker, K. L. (2007). Television Viewing Is Associated With Prevalence of Metabolic Syndrome in Hispanic Elders. Diabetes Care, 30, 694-700.

Yang, H., Schnall, P., Jáuregui, M., Su, T., y Baker, D. (2006). Exceso de trabajo e Hipertensión Arterial. Revista Hypertension, 48, 1-7.

Fecha de recepción: 17 de junio del 2008.

Fecha de aceptación: 23 de octubre del 2008.

Fecha de publicación: 31 de julio del 2009. 